\title{
Coupling System for Ultra Precision Machining
}

\author{
Eike Foremny, Christian Schenck and Bernd Kuhfuss \\ Bremen Institute for Mechanical Engineering, MAPEX Center for Materials and Processing, University of Bremen, Bremen 28209, \\ Germany
}

\begin{abstract}
The mass production of plastic parts containing micro structures in optical quality requires molds with corresponding surface quality. These micro structured molds can be produced by UPC (ultra precision cutting). To produce a high surface quality the ultra precision machine spindles must be well balanced. It is not sufficient to balance the tools and the spindle separately. They have to be balanced while assembled to ensure the required balance quality grade better than G0.4. One possibility to decrease setup times and increase the quality of the spindle balancing is to enhance the unbalance induced vibrations and thus allowing better unbalance detection. In this paper a coupling system between the spindle and machine frame based on flexure joints is presented. The experimental setup provides two states: operational and setup. The operational state provides a high stiffness, whereas the setup state allows the spindle to vibrate along one degree of freedom, enhancing the vibrations created by the unbalance. The system is analyzed concerning its ability to restore the defined conditions in the operational state as well as its capability to enhance the detection of unbalances in the setup state.
\end{abstract}

Key words: Optical surfaces, high precision balancing, coupling system.

\section{Introduction}

An increase in productivity is becoming more and more important in micro and ultra precision machining. The mass production of plastic parts with micro structures, e.g. camera optics or retro reflectors, relies on the quality of the used molds [1]. Using UPC (ultra precision cutting) freeform molds with good surface quality can be created [2].

The setup time in UPC typically takes up to $30 \%$ of the overall process time. In the UPC as e.g. milling with fly-cutter approximately $70 \%$ of the setup time is used for balancing the spindle and calibrating the tool. It is a manual process [3-6]. The spindle speed is incrementally increased until the measurement system detects the unbalance. After stopping the spindle and applying a counterweight the process is restarted until the desired spindle speed and balance state is reached. Due to the multiple start-up and run-down sequences the balancing process is very time consuming.

One possibility to reduce the setup times is to balance the spindle at lower speeds with improved

Corresponding author: Eike Foremny, M.Sc., research field: ultra precision spindle balancing. resolution of the detectable unbalance level at the same time. This could be achieved by changing the dynamic properties of some machine parts as e.g. the connection between the spindle and the base (Fig. 1). But afterwards the origin state with respect to the position as well as the dynamic properties has to be restored.

This approach could be realized using a coupling system which is temporarily able to decrease the stiffness between spindle and base. It could mechanically amplify the vibrations generated by the unbalance thus enabling the balancing at lower speeds. Furthermore, it could lead to better balanced spindles due to the ability to detect smaller unbalances. Additionally, this could lead to higher spindle speeds or better surface quality and establish the first step towards an automatic balancing system for UPC spindles.

\section{Experimental Apparatus}

\subsection{Experimental Coupling System}

The purpose of the presented coupling system is to create a variable connection between the spindle and the machine base. It should allow for machining as well 


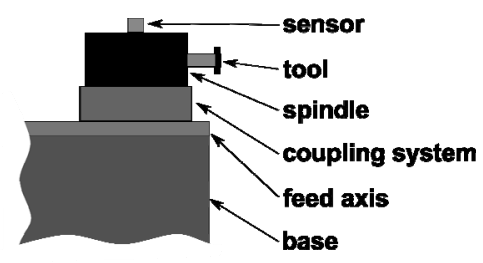

Fig. 1 Machine tool with the proposed coupling system.

as increase the ability to detect smaller unbalances. The experimental coupling system can take two states.

In the operational state the top plate is connected with the bottom plate via the flexure joints as well as the coupling joints (Fig. 2). The purpose of this state is to create a stiff connection between the spindle and the base to enable machining.

In the setup state only the flexure joints connect the top and bottom plate. This constellation locks all degrees of freedom except the x-direction. Due to the flexure joints the top plate is able to vibrate predominantly in $\mathrm{x}$-direction but always returns roughly to the original position.

To switch from the operational to the setup state the two coupling joints are spread and the coupling sphere is released from the top plate. The spreading is realized by the set screw, which is connected to both coupling joints (Fig. 3). To switch back into the operational state the coupling joints are pulled towards each other, reconnecting the coupling spheres with the top plate. The set screw is equipped with a force sensor to measure the manually applied coupling force.

\subsubsection{Fitting of the Casting Spheres}

To create a releasable connection between the flexure joints and the top plate the coupling spheres are used. The coupling spheres are initially fitted into the top plane by casting. The polymer/metal mixture Moglice was used as adjustment coating. It is claimed by the producer to create surface formings with accuracy better than $1 \mu \mathrm{m}$ while providing a Young's modulus of about $10 \mathrm{kN} / \mathrm{mm}^{2}$. The spheres are mounted on the flexure bearing and then fitted into the inlet (Fig. 4).

\subsection{Measurement System}

The force is measured by a piezoelectric force sensor in combination with an amplifier. The sensor has a sensitivity of $-4.3 \mathrm{pC} / \mathrm{N}$ and a maximal load of $62 \mathrm{kN}$. A Kistler 5007 charge amplifier with a long hold time is used. Due to the working principle of the amplifier the measurement is not accurate at low frequencies or even steady forces, resulting in a force measurement uncertainty of about $\pm 50 \mathrm{~N}$.

Two measurement systems are used to determine the difference between the dynamic properties of the two states as well as the recoverability of the coupling mode. The position error along the $\mathrm{x}$ axis is examined with a laser interferometer (ML10 Renishaw) which has a resolution of $1 \mathrm{~nm}$ at $5 \mathrm{kHz}$. The measurement noise in this setup is about $50 \mathrm{~nm}$ (Fig. 5). The dynamic properties of the coupling system are measured using a Bruel \& Kjaer accelerometer with a sensitivity of 1 $\mathrm{mV} / \mathrm{ms}^{2}$ and a frequency range from $2-7,000 \mathrm{~Hz}$. The coupling system is excited with an impulse hammer.

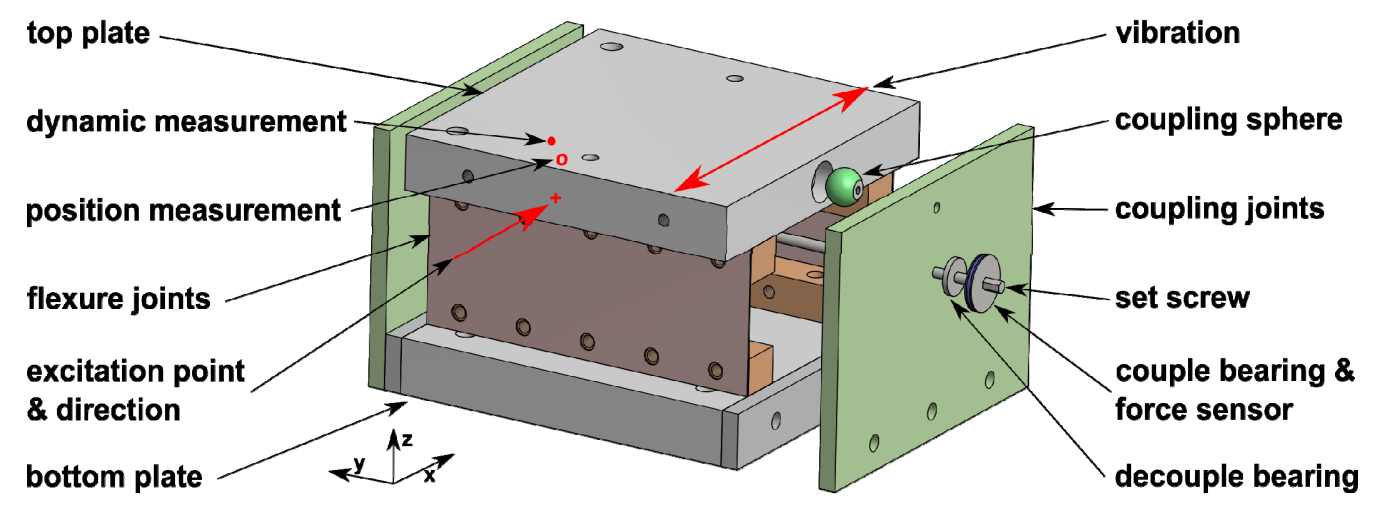

Fig. 2 Experimental coupling system with coupling components. 


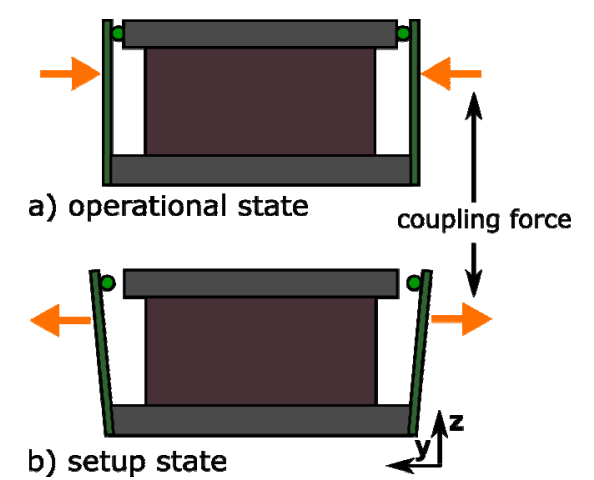

Fig. 3 System coupling states and direction of coupling forces.

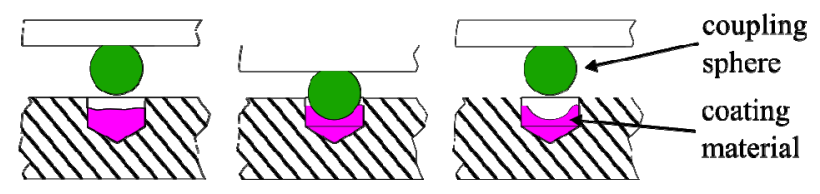

Fig. 4 Casting of the sphere into the inlet.

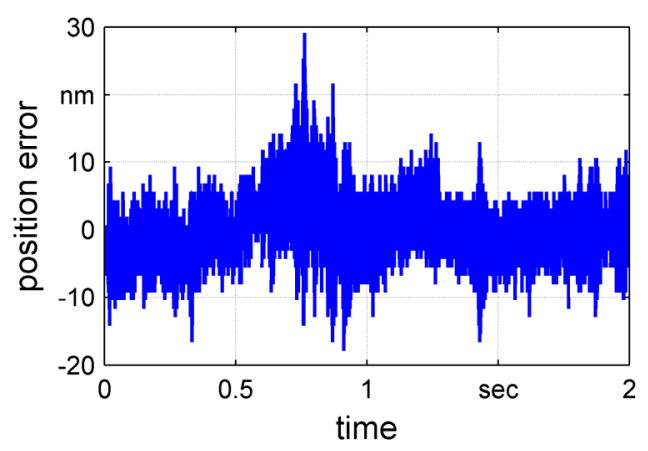

Fig. 5 Noise in the laser interferometer measurements.

\section{Experiments}

In order to determine the capability of the presented coupling system two groups of experiments are conducted. First the dynamic properties of the setup in the operational as well as the setup state are evaluated. Second the position recovery along the x-axis of the operational state is determined.

\subsection{Dynamic Properties}

In order to determine the dynamic properties of the coupling system two experiments are conducted. The first one evaluates the difference between the two coupling states in terms of the frequency response. The second one examines the changes in the frequency response after recoupling.

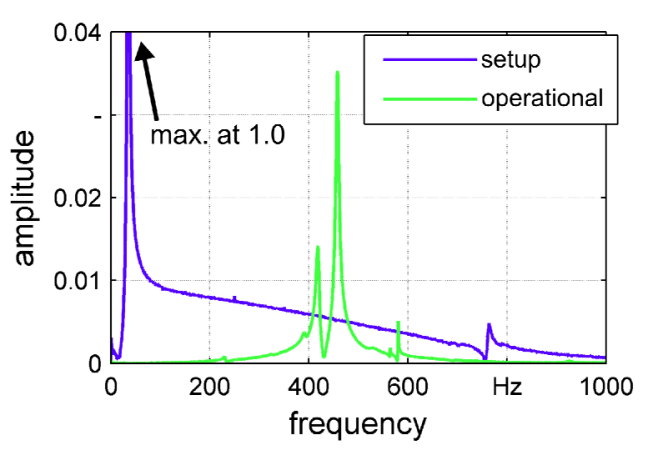

Fig. 6 Dynamic properties of the two states.

Both coupling modes are excited with an impulse hammer with similar force and at similar positions (Fig. 2). The frequency response is normalized to the excitation impulse and plotted in the frequency domain (Fig. 6). Three major changes can be stated. Firstly, the maximum amplitude increases by factor 28.3 between the operational and setup state. Secondly, in contrast to the operational state the frequency with the highest amplitude is shifted from $458 \mathrm{~Hz}$ to $35 \mathrm{~Hz}$ in the setup state. Thirdly, only two eigenfrequencies of the three remain in the setup state.

The coupling force in the operational state is varied over a wide range to investigate the repeatability of the dynamic behaviour of the experimental coupling system. The first and second eigenfrequencies are nearly consistent over the tested coupling force range. In contrast the third eigenfrequency changes over 500 $\mathrm{Hz}$ (Fig. 7). Furthermore, it can be seen that there is a proportional dependency between the force and the third eigenfrequency of the coupling system. The ratio is approximately $0.06 \mathrm{~Hz} / \mathrm{N}$. Since the third eigenfrequency changes strongly with the coupling force, it can be assumed that it originates from the top plate or the coupling elements.

When the coupling force is kept as constant as possible the overall frequency stability is good. Fig. 8 shows five frequency response functions with coupling forces between 2,550 $\mathrm{N}$ and 2,620 N. The coherence function is always well above 0.8 which validates a clear causality between excitation and response. But as expected the third eigenfrequency at around $530 \mathrm{~Hz}$ is 


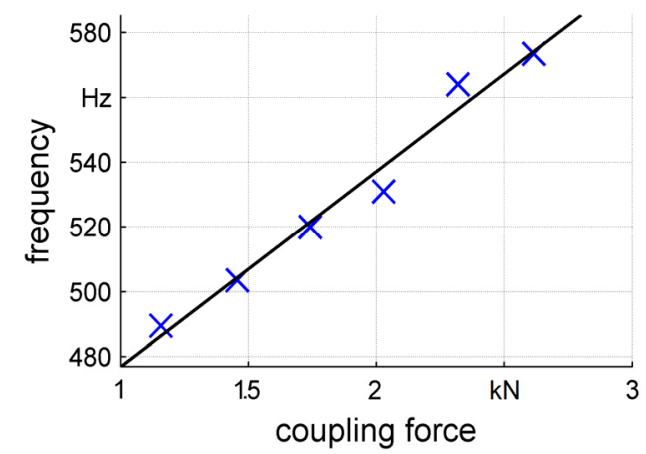

Fig. 7 Shift of the third. eigenfrequency as a function of the coupling force.

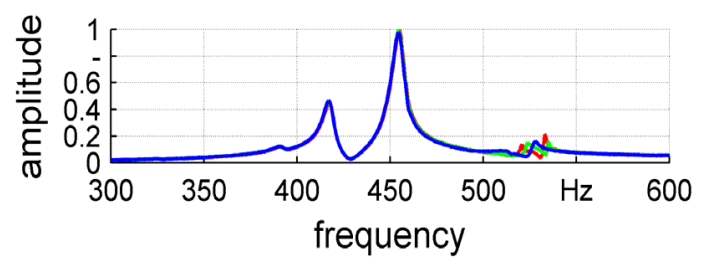

Fig. 8 Eigenfrequency recovery.

very inconsistent and changes about $10 \mathrm{~Hz}$. This can be explained by the deviations in the coupling force.

\subsection{Position Repeatability}

In addition to matching dynamic properties the original position has to be recovered accurately in order to speed up of the setup times. From the experiences in the dynamic evaluation the position experiments are started over a wide range of coupling force as well. It is varied from $1,550 \mathrm{~N}$ to $2,750 \mathrm{~N}$. Additionally, every coupling force is adjusted from the rising and from the falling side. It can be seen that the recovered position is linearly dependent on the coupling force (Fig. 9). The linear regression is about $-8 \mathrm{~nm} / \mathrm{N}$. Furthermore, it has an impact whether the force is adjusted by increasing or decreasing the set screw torque. At present it is not quite clear whether the observed difference between the two is an effect from the applied torque, which could move the coupling system as a whole, or from the uneven elastic deformation of the spheres and inlets.

It is intended to keep the coupling force equal in all operational states. The target coupling force is $2.6 \mathrm{kN}$, always approached from the bottom. First the current $\mathrm{x}$ position is set as reference point. Then the system is decoupled by spreading the two flexure bearing until the contact between the spheres and the coating material is lost. Finally, the coupled mode is recovered by applying the pre-defined force to both flexure bearings. The whole sequence is measured in one recording. The quality of the recovery is determined by the position difference between the two coupled states. It is possible to re-adjust the position along the $\mathrm{x}$-axis with an accuracy of $-12 \mathrm{~nm} \pm 84 \mathrm{~nm}$. Due to the uncertainty of the measurement tools the trend from the coarse measurement is no longer clearly visible (Fig. 10). To further determine the position accuracy more precise measurement systems e.g. capacitive distance sensors are needed.

\subsection{Spindle Balancing}

In order to test the capability of the coupling system to improve the balance detection an exemplary balancing process was conducted. A spindle, mounted

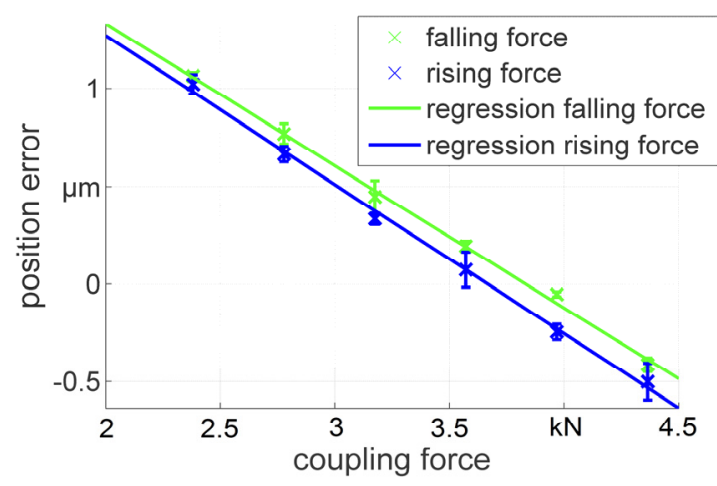

Fig. 9 Coupled position at different coupling forces.

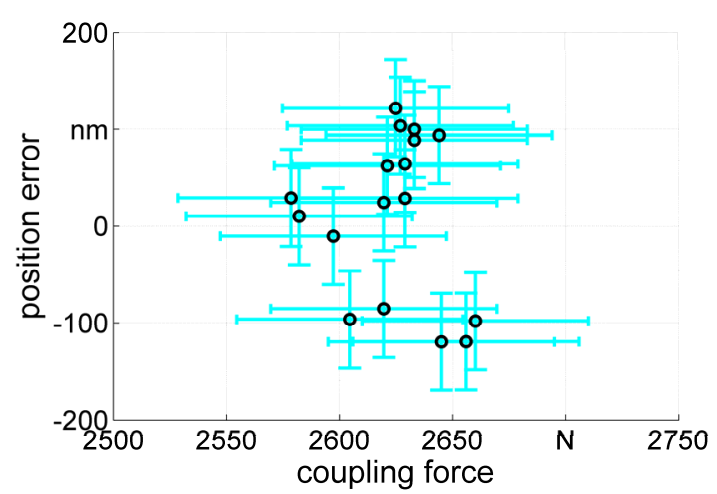

Fig. 10 Position recoveries with estimated uncertainty. 


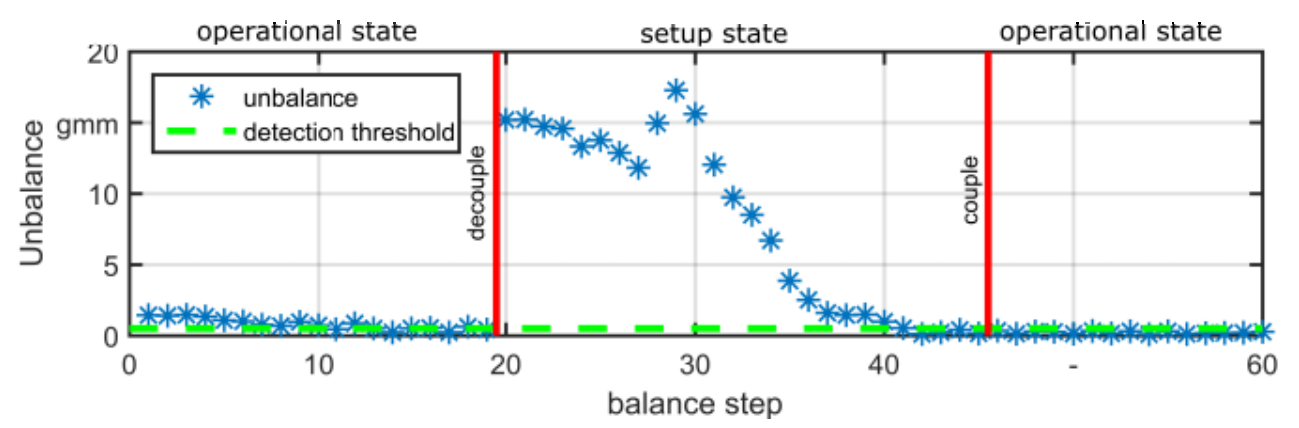

Fig. 11 Balance process supported by the coupling system.

on the coupling system, was balanced in the operational state until the detection threshold from the unbalance detection system was reached. By switching the coupling system into the setup state it was possible to increase the detection and consequently the reduction of the remaining unbalance by a factor of 75 . Afterward the system was switched back into the operational state (Fig. 11).

\section{Conclusion and Outlook}

In this paper a high precision coupling system, which can facilitate the setup of ultra precision machines, is analysed.

The experimental coupling system presented in this paper is able to change between the operational and the setup state. The operational state features a high stiffness and position recoverability and can be used for machining. In the setup state the spindle is able to vibrate along one axis to amplify vibrations induced by unblanaces.

The recovery of the overall frequency response function in the operational state is good. The third eigenfrequency can only be recovered with an accuracy of $10 \mathrm{~Hz}$. It is shown that the position recovery between two operational states is linear dependent on the coupling force. The recovery of one position is possible in the range of $\pm 100 \mathrm{~nm}$.

In the setup state the experimental coupling system has a stiffness which is over 28 times lower than in operational state. Furthermore, the eigenfrequency of highest amplitude decreases from $458 \mathrm{~Hz}$ to $35 \mathrm{~Hz}$. Additionally, the change in the resonance frequency can be useful for balancing purposes as well. Because the speed at which the spindle is balanced can be lower.

It is shown that the presented coupling system is able to significantly improve the balance detection and thus enhance the resulting balance quality of the spindle.

Since it is difficult to measure the coupling force further works will try to determine it indirectly. It is shown that the third eigenfrequency is linear dependent on the coupling force. Therefore, the frequency of the eigenfrequency may be used to derive the coupling force. Since the frequency can be measured accurately it could be used to recovery the position with higher precision.

In future works, it is planned to merge the presented coupling system with a variable stiffness system. This system is able to change its stiffness and eingenfrequencies continuously between the two states: operational and setup. This allows to combine the advantages of the two systems. On the one hand, the high position reproducibility and on the other the possibility to adjust the eigenfrequency and stiffness to the requirements of the current process.

\section{Acknowledgements}

The authors would like to thank the German Research Foundation (DFG) for funding this work as a part of the Research Unit FOR1845 "Ultra-Precision High Performance Cutting".

\section{References}

[1] Bäumer, S. 2010. "Handbook of Plastic Optics." Wiley-VCH Publ. Co.

[2] Brecher, C., Lindemann, D., Merz, M., Wenzel, C., and 
Preuß, W. 2013. "Freeform Machining of Molds for Replication of Plastic Optics." Fabrication of Complex Optical Components. From mold design to product, Berlin Heidelberg: Springer, 21-52.

[3] Darlow, M. 1989. Balancing of High-Speed Machinery. New York: Springer-Verlag, 53-66.

[4] Schneider, H. 1992. "Auswuchttechnik", VDI-Verlag, 95-128.
[5] Federn, K. 2011. "Auswuchttechnik", Auswuchttechnik, Klassiker der Technik: Band 1: Allgemeine Grundlagen, Meßverfahren und Richtlinien, Klassiker der Technik, Springer-Verlag Berlin Heidelberg.

[6] B. Knapp, et al. 2011. "The importance of spindle balancing for the machining of freeform optics." Presented at the ASPE spring topical meeting on structured and freeform surfaces, USA. 